\title{
Impact of interstitial lung disease on surgical morbidity and mortality for lung cancer: Analyses of short-term and long-term outcomes
}

\author{
Masako Chiyo, MD** \\ Yasuo Sekine, MD ${ }^{\mathrm{a} *}$ \\ Takekazu Iwata, MD \\ Koichiro Tatsumi, MD \\ Kazuhiro Yasufuku, MDa \\ Akira lyoda, $M D^{a}$ \\ Mizuto Otsuji, MD \\ Shigetoshi Yoshida, MD \\ Kiyoshi Shibuya, MD ${ }^{\mathrm{a}}$ \\ Toshihiko lizasa, $M D^{a}$ \\ Yukio Saitoh, MD \\ Takehiko Fujisawa, MDa
}

From the Departments of Thoracic Surgery $^{\mathrm{a}}$ and Respirology, ${ }^{\mathrm{b}}$ Graduate School of Medicine, Chiba University, Chiba, Japan.

This study was supported by grant-in-aid for scientific research (No. 12671299) from the Japanese Ministry of Education, Culture, Sports, Science and Technology.

Received for publication July 22, 2002; revisions requested Oct 11, 2002; revisions received Feb 17, 2002; accepted for publication March 24, 2003.

Address for reprints: Yasuo Sekine, MD, Department of Thoracic Surgery, Graduate School of Medicine, Chiba University, 1-8-1 Inohana, Chuo-ku, Chiba 260-8670, Japan (E-mail: yasuo-sekine@umin.ac.jp).

*M.C. and Y.S. contributed equally to this work and are considered co-first authors.

J Thorac Cardiovasc Surg 2003;126:1141-6

Copyright (C) 2003 by The American Association for Thoracic Surgery

0022-5223/2003\$30.00+0

doi:10.1016/S0022-5223(03)00791-8
Background: This study investigated postoperative morbidity, mortality, and the long-term survival for patients with lung cancer who have interstitial lung diseases.

Methods: A retrospective chart review of 931 patients with lung cancer who underwent pulmonary resection at Chiba University Hospital between 1990 and 2000 was undertaken. Interstitial lung disease was defined by medical history, physical examination, and abnormalities compatible with bilateral lung fibrosis on chest computed tomography or high-resolution computed tomography (36 patients: $3.9 \%$, interstitial lung diseases group). The remaining 895 patients $(96.1 \%)$ were categorized as non-interstitial lung disease group.

Results: The incidence of postoperative pneumonia and acute or exacerbation of interstitial pneumonia was higher in the interstitial lung disease group (all $P<.05$ ). Thirty-day mortality was statistically equivalent between the interstitial lung disease and the non-interstitial lung disease groups $(P=.30)$. The 5 -year overall survivals were $62.5 \%$ (non-interstitial lung disease) and $35.6 \%$ (interstitial lung disease). Respiratory failure was the second main cause of death after the recurrence of primary cancer in the interstitial lung disease group. The risk factors for long-term mortality were interstitial lung diseases, advanced pathologic stage, male sex, high age, and positive smoking history (all $P<.05$ ).

Conclusions: Interstitial lung disease was a risk factor for developing postoperative morbidity and mortality and poor long-term survival due to the occurrence of respiratory failure.

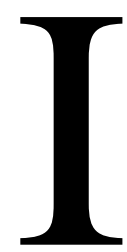

nterstitial lung disease (ILD), such as idiopathic pulmonary fibrosis (IPF), interstitial pneumonia due to collagen vascular diseases, and occupational lung diseases, have been widely known to be associated with lung cancer. Several environmental hazards, such as cigarette smoking and occupational exposures, are proposed as common risk factors for ILD. ${ }^{1,2}$ When such ILDs are associated with lung cancer, the existence of ILD becomes an obstacle for surgical treatment due to high postoperative pulmonary complications and mortality. However, the influences of the existence of ILD as a complication on 
morbidity, mortality, and long-term survival after pulmonary resection for lung cancer has not been well studied. Therefore, the purpose of this study was to identify the incidence of postoperative complications and mortality after lung cancer surgery in the presence of ILD and to clarify the impact of preoperative existence of ILD on the long-term survival after pulmonary resection for lung cancer.

\section{Patients and Methods \\ Patients}

The medical records of 931 consecutive patients with lung cancer who underwent pulmonary resection between January 1990 and March 2000 at Chiba University Hospital were retrospectively reviewed. Data were collected from our institutional cancer registry database and from patient follow-up visits in the physician's office. The records contain detailed preoperative patient characteristics, disease status, operative procedures, postoperative complications, pathologic diagnosis, neoadjuvant and adjuvant therapies, and follow-up data. These data were collected at the time of hospital discharge and from outpatient charts in a prospective basis. Preoperative chest conventional or high-resolution computed tomography (HRCT) was evaluated to examine not only the extent of cancer but also the existence of ILD in all cases. ILD was defined by medical history, physical examination, and abnormalities compatible with bilateral lung fibrosis on chest computed tomography (CT) or HRCT, such as peripheral reticular opacities. When the chest CT, reviewed by radiologists, revealed such abnormalities, patients were referred to pulmonologists and the existence of ILD was evaluated preoperatively. Non-ILD and ILD groups consisted of $895(96.1 \%)$ and 36 (3.9\%) patients, respectively, of all enrolled patients. The ILD group included 26 patients with IPF, 5 with pneumoconiosis, and 5 with connective tissue diseases (4 rheumatoid arthritis and 1 Sjögren syndrome). Three patients received steroid therapy before operation. Patients with severely symptomatic ILD who had diffuse IP on chest CT were usually judged inoperable. All identified IPF patients showed radiologically localized changes, which were restricted in the bilateral lung basal segmental areas or lower lobes and confirmed by chest CT.

Preoperative evaluation for all patients otherwise included a detailed medical history, physical examination, blood and urine examinations, and a 12-lead electrocardiogram. Incentive spirometry and nebulizing by distilled water with or without bronchodilator were routinely encouraged for enhancing lung expansion and airway clearance at least for 2 weeks before and after surgery. Neoadjuvant or adjuvant therapy, which was platinum agentbased chemotherapy and radiation, was performed mainly for the patients who had locally advanced disease or multiple nodal involvement, or who underwent incomplete resection. The postoperative morbidities included bacterial pneumonia (confirmed by infiltrative shadows on chest radiograph, positive sputum culture, body temperature $\geq 37.5^{\circ} \mathrm{C}$, and white blood cell count $>10,000$ / $\mu \mathrm{L}$ ), interstitial pneumonia (aggravation of dyspnea on exertion, deterioration of arterial blood gas analysis, and diffuse interstitial abnormalities compatible with acute interstitial pneumonia [AIP] in the non-ILD group or exacerbation of IP in the ILD group on chest radiograph and CT), mechanical ventilation for more than 3 days, bronchial stump dehiscence, empyema (positive bacterial infection of pleural effusion), tracheostomy (scheduled at the time of operation and postoperative emergency procedure), and postoperative home oxygen therapy (HOT) for patients with $\mathrm{PaO}_{2}$ less than $55 \mathrm{~mm} \mathrm{Hg}$ at rest or less than $60 \mathrm{~mm} \mathrm{Hg}$ on exercise at the time of hospital discharge.

After discharge from the hospital, patients visited our outpatient clinic regularly every 1 to 6 months, unless tumor recurred or the patient had any health problems. When patients returned to the referral doctors or were introduced to other doctors, the patients' condition and causes of death (if patients died) were confirmed by mail or telephone to the patient, patient's family, or referral doctors every 2 years. The follow-up was completed in 908 of 931 patients through March 2001 (with loss of follow-up data of 23 non-ILD patient) and the median follow-up was 43.8 months. The overall survival was analyzed for each group of the patients.

\section{Statistical Analysis}

Data were analyzed with StatView version 5.0 software (Statistical Analysis Systems; Cary, NC). To compare the differences between the ILD and non-ILD groups, a Mann-Whitney $U$ test was used to analyze for continuous variables, and the $\chi^{2}$-square test or Fisher exact test to analyze for categorical variables. The survival curves were estimated with the Kaplan-Meier method, and the difference in survival times between the 2 groups was calculated by the log-rank test. The Cox proportional hazard model was conducted for identifying prognostic factors.

\section{Results}

\section{Preoperative Patient Characteristics}

Preoperative patient characteristics are summarized in Table 1. Male sex was more predominant in the ILD group than in the non-ILD. Although smoking history was significantly more prevalent in the ILD than the non-ILD $(P<.001)$, smoking index was not statistically significant between the 2 groups. Comparing pulmonary function tests and arterial blood gas values between the 2 groups, percent forced vital capacity (\%FVC), forced expiratory volume in 1 second $\left(\mathrm{FEV}_{1}\right), \%$ predicted $\mathrm{FEV}_{1}$, and $\mathrm{FEV}_{1} / \mathrm{FVC}$ in patients with ILD were significantly lower than those in patients without ILD. There were similar number of patients who received neoadjuvant chemotherapy or radiation therapy between the 2 groups.

Perioperative characteristics are listed in Table 2. Pneumonectomy was not performed in the ILD group. Because tumor size was relatively large (maximal diameter: $4.5 \pm$ $2.7 \mathrm{~cm}$ ) and pulmonary function was preserved in the ILD group, limited pulmonary resection (segmentectomy or partial lung resection) was not a preferred method compared with the non-ILD group. Therefore, the selection of operative methods was similar between the groups. The distributions of histologic types and pathologic stages were also similar between the 2 groups. There were no differences in the number of adjuvant treatments between the 2 groups. 


\section{Postoperative Morbidity and Mortality}

Postoperative pulmonary complications and mortality are summarized in Table 3. The frequencies of all specific pulmonary complications were significantly higher in the patients with ILD as compared with the patients without ILD. In particular, 27 patients had postoperative AIP in the non-ILD group or deterioration of IP in the ILD group. The incidence in the ILD group was significantly higher than that in the non-ILD group $(P<.0001)$. Although they received postoperative steroid pulse therapy (intravenous injection of methylprednisolone 0.5 to $1 \mathrm{~g}$ for 3 days) or high dose of prednisone (30 to $60 \mathrm{mg} / \mathrm{d}$ ), 4 patients ( 1 in the non-ILD and 3 in the ILD) died of uncontrollable IP in hospital. Thirty-day mortality in patients with ILD was equivalent to that of patients without $\operatorname{ILD}(P=.30)$. The causes of 30-day mortality in the non-ILD group were as follows: empyema with bronchial fistula, 2; bacterial pneumonia, 1; interstitial pneumonia, 1; empyema, 1; myocardial infarction, 1; massive bleeding, 1; and brain infarction, 1. Deterioration of IP was observed in 1 case in the ILD group.

\section{Survival Analyses}

Figure 1 shows overall survival after the the surgery. The cumulative survivals at 3 and 5 years were $71.0 \%$ and $62.5 \%$ in the non-ILD group and $41.5 \%$ and $35.6 \%$ in the ILD group, respectively $(P<.0001)$.

The causes of deaths are shown in Table 4. Respiratory failure due to bacterial pneumonia, AIP, and deterioration of IP was the second most frequent cause of death after the recurrence of primary cancer in the ILD group (33.3\%) and was seen in a higher proportion in the ILD group than in the non-ILD group $(P=.0028)$. The investigation of long-term respiratory complications in 35 patients with ILD who survived more than 30 days revealed that 7 patients had immediate deterioration of IP. Two of them died between 30 and 60 days and 5 patients gradually progressed. One patient had bacterial pneumonia with bronchial fistula and died at the 33 days postoperatively. In 4 patients, a gradual progression of fibrosis was seen, despite no initial change of IP. One patient had no deterioration of IP but frequent pneumonia. There were no changes in the respiratory condition in 22 patients $(22 / 35,62.9 \%)$ during the observation period.

We also attempted to clarify risk factors for long-term overall mortality (Table 5). ILD, male sex, higher age, positive smoking history, and pathologic stages III and IV (all $P<.05$ ) were identified as independent risk factors according to Cox's proportional hazard model.

\section{Discussion}

It has been reported that ILD including IPF, asbestosis, and silicosis are associated with a high risk of lung cancer. ${ }^{1,3}$
TABLE 1. Preoperative characteristics of lung cancer patients

\begin{tabular}{|c|c|c|c|}
\hline Characteristics & $\begin{array}{c}\text { Non-ILD } \\
(n=895)\end{array}$ & $\begin{array}{c}\text { ILD } \\
(n=36)\end{array}$ & $P$ value \\
\hline \multicolumn{4}{|l|}{ Gender } \\
\hline Male & $599(66.9 \%)$ & $32(88.9 \%)$ & .006 \\
\hline Female & 296 & 4 & \\
\hline Age (year)* & $63.1 \pm 10.1$ & $65.5 \pm 7.0$ & .200 \\
\hline BMI $\left(\mathrm{kg} / \mathrm{m}^{2}\right)^{*}$ & $22.2 \pm 3.0$ & $21.5 \pm 2.7$ & .280 \\
\hline PSH $(\%)$ & $614(68.6 \%)$ & $35(97.2 \%)$ & $<.0001$ \\
\hline $\begin{array}{l}\text { Smoking index* (pack- } \\
\text { year) }\end{array}$ & $33.6 \pm 33.0$ & $49.7 \pm 25.2$ & .216 \\
\hline \multicolumn{4}{|c|}{ Pulmonary function tests* } \\
\hline FVC (L) & $2.95 \pm 0.74$ & $2.74 \pm 0.61$ & .1759 \\
\hline$\% F V C(\%)$ & $97.3 \pm 17.3$ & $84.9 \pm 17.4$ & .0001 \\
\hline $\mathrm{FEV}_{1}(\mathrm{~L})$ & $2.22 \pm 0.59$ & $1.96 \pm 0.52$ & .0136 \\
\hline$\% \mathrm{FEV}_{1}(\%)$ & $84.4 \pm 18.8$ & $68.7 \pm 17.1$ & $<.0001$ \\
\hline $\mathrm{FEV}_{1} / \mathrm{FVC}(\%)$ & $75.9 \pm 9.8$ & $70.8 \pm 12.8$ & .0191 \\
\hline $\mathrm{PaO}_{2}(\mathrm{~mm} \mathrm{Hg})$ & $87.8 \pm 9.6$ & $85.4 \pm 9.8$ & .0910 \\
\hline $\mathrm{PaCO}_{2}(\mathrm{~mm} \mathrm{Hg})$ & $40.9 \pm 3.2$ & $40.2 \pm 3.4$ & .1360 \\
\hline Neoadjuvant therapy & $14(1.6 \%)$ & $1(2.8 \%)$ & .449 \\
\hline chem/rad/both & 8/5/1 & $1 / 0 / 0$ & \\
\hline
\end{tabular}

$B M I$, Body mass index, PSH, positive smoking history; chem, chemotherapy; rad, radiation; both, chemotherapy and radiation.

${ }^{*}$ Data are presented as mean $\pm S D$.

Common characteristics of these diseases are male sex, high age, and heavy cigarette smoking. As these patients have pulmonary impairment and poor reserve of cardiopulmonary function, many patients do not match operative indications and need palliative care. In our study, lung cancer with ILD was significantly more frequently found in men and those with positive smoking history.

Although pulmonary function was significantly lower in the ILD group, the PFT data showed that patients had enough pulmonary reserve for operation. Flaherty and Martine $\mathrm{z}^{4}$ reported that pulmonary function testing can provide an estimate of histologic severity but not a definitive quantification of histologic fibrosis or inflammation. Martinod and colleagues ${ }^{5}$ reported pulmonary functions of 20 ILD patients who underwent pulmonary resection for lung cancer. They had well-preserved pulmonary functions similar to our results. Doherty and coworkers ${ }^{6}$ reported that heavy smoking patients with IPF (most of them were men) tended to preserve lung volumes and that emphysema due to smoking could explain the relative volume preservation. In this study, IPF or IP due to collagen vascular diseases were limited in the basal segments or lower lobes preoperatively, and patients with ILD had higher prevalence rate of positive smoking history than those without ILD. This may be one of the reasons for $\mathrm{FEV}_{1} / \mathrm{FVC}$ reduction due to emphysematous change. Therefore, it is believed that PFT did not reflect the severity of ILD.

ILD had an adverse effect on short- and long-term survivals. Postoperative deterioration of IP necessitating me- 
TABLE 2. Perioperative characteristics of lung cancer patients

\begin{tabular}{|c|c|c|c|}
\hline Characteristics & Non-ILD $(n=895)$ & ILD $(n=86)$ & $P$ value \\
\hline Operation methods & & & .216 \\
\hline Pneumonectomy & $62(6.9 \%)$ & $0(0 \%)$ & \\
\hline Lobectomy & $784(87.6 \%)$ & $33(91.7 \%)$ & \\
\hline Segmentectomy/partial resection & $49(5.5 \%)$ & $3(8.3 \%)$ & \\
\hline Tumor size $(\mathrm{cm})$ (maximal diameter)* & $3.5 \pm 3.4$ & $4.5 \pm 2.7$ & .082 \\
\hline Histologic diagnoses & & & .142 \\
\hline $\mathrm{Ad} / \mathrm{Sq} / \mathrm{Sm} / 0$ th & $530 / 276 / 13 / 76$ & $18 / 14 / 2 / 2$ & \\
\hline$\%$ & $59.2 / 30.8 / 1.5 / 8.5$ & $50 / 38.8 / 5.6 / 5.6$ & \\
\hline Pathologic $\mathrm{T}$ status & & & .226 \\
\hline $\mathrm{T} 1 / 2 / 3 / 4$ & $349 / 267 / 96 / 178$ & $8 / 13 / 7 / 8$ & \\
\hline$\%$ & $39 / 29.8 / 10.7 / 19.9$ & $22.2 / 36.1 / 19.4 / 22.2$ & \\
\hline Pathologic nodal status & & & 689 \\
\hline N0/1/2/3 & $515 / 133 / 196 / 33$ & $17 / 5 / 11 / 2$ & \\
\hline$\%$ & $57.5 / 14.9 / 21.9 / 3.7$ & 47.2/13.9/30.5/5.6 & \\
\hline Pathologic staging & & & .342 \\
\hline I/II/III/IV & 387/118/324/48 & $10 / 4 / 18 / 3$ & \\
\hline$\%$ & $43.2 / 13.2 / 36.2 / 5.4$ & $27.8 / 11.1 / 50 / 8.3$ & \\
\hline Adjuvant therapy & $177(19.8 \%)$ & $4(11.1 \%)$ & .281 \\
\hline (chem/rad/both) & $114 / 61 / 2$ & $4 / 0 / 0$ & \\
\hline
\end{tabular}

$A d$, Adenocarcinoma; $S q$, squamous cell carcinoma; $S m$, small cell carcinoma; Oth, other histology; chem, chemotherapy; rad, radiation; both, chemotherapy and radiation.

*The size was measured from the resected specimen.

TABLE 3. Postoperative respiratory complications and mortality

\begin{tabular}{lccr}
\hline & $\begin{array}{c}\text { Non-ILD } \\
(\boldsymbol{n}=\mathbf{8 9 5 )}\end{array}$ & $\begin{array}{c}\text { ILD } \\
(\boldsymbol{n}=\mathbf{3 6})\end{array}$ & $\boldsymbol{P}$ value \\
\hline Pneumonia (\%) & $57(6.4 \%)$ & $6(16.7 \%)$ & .0295 \\
IP & $18(2.0 \%)$ & $9(25 \%)$ & $<.0001$ \\
Prolonged mech vent & $27(3.0 \%)$ & $5(13.9 \%)$ & .0061 \\
HOT & $2(0.2 \%)$ & $4(11.1 \%)$ & $<.0001$ \\
Tracheostomy & $75(8.4 \%)$ & $12(33.3 \%)$ & $<.0001$ \\
$\quad$ Scheduled & $38(50.7 \%)$ & $4(33.3 \%)$ & \\
$\quad$ Emergency & $37(49.3 \%)$ & $8(66.7 \%)$ & \\
30-day mortality & $8(0.9 \%)$ & $1(2.8 \%)$ & .300 \\
\hline
\end{tabular}

$\overline{I P}$, Interstitial pneumonia; Prolonged mech vent, mechanical ventilation $\geq 3$ days; HOT, home oxygen therapy; SVT, supraventricular tachycardia.

chanical ventilation and tracheostomy was a major cause of death and occurred more frequently in ILD patients than in non-ILD patients. We also clarified that patients with ILD had worse overall survival than those without ILD. This may be because of subsequent respiratory failure due to postoperative AIP or exacerbation of ILD. Another explanation is that ILD may stimulate rapid tumor progression. Matsushima and colleagues ${ }^{7}$ reported that lung cancers originated in the border area of honeycombing, and they suggested a close relationship of epithelial changes and carcinogenesis in ILD patients. As biological characteristics of cancer in patients with ILD are unknown, further investigation is needed on this issue.

In terms of treatment for postoperative IP, corticosteroids are often used as a first-line treatment with regimens similar to those in primary IPF and IP due to collagen vascular diseases (CVD). Although results of corticosteroid treatment were highly variable and optimal therapy has not been established, ${ }^{8,9}$ rheumatoid arthritis-associated ILD appeared to have a better prognosis than IPF in most studies. ${ }^{10}$

In terms of prognosis, the prognostic difference among types of ILD after pulmonary resection is unknown. The prognosis of IPF is generally very poor; the overall mean survival was reported to range between 3 and 6 years. ${ }^{11}$ On the other hand, the prognosis of IP due to CVD is relatively better than that of IPF, but it depends on the histology. ${ }^{12}$ It cannot be denied that ILD itself affected the prognosis of lung cancer patients. Battafarano and colleagues ${ }^{13}$ reported that comorbidity had a strong impact on survival in patients with stage I non-small cell lung cancer. In this study, only 22 ILD patients could keep stable respiratory conditions over an extended length of time. Because of such a small number of ILD patients, we could not reach the postoperative prognostic difference depending on the type of ILD, although we have the impression that CVD-associated IP and pneumoconiosis patients may maintain stable conditions unless severe postoperative pulmonary complications occur.

The Cox proportional hazard model revealed that ILD, advanced pathologic stage (III and IV), male sex, high age, and positive smoking history were the significant risk factors for long-term mortality. Except for ILD, these factors are closely related and affect mortality. In our study, we demonstrated that ILD exacerbated long-term survival in addition to these other factors. Therefore, when we decide 


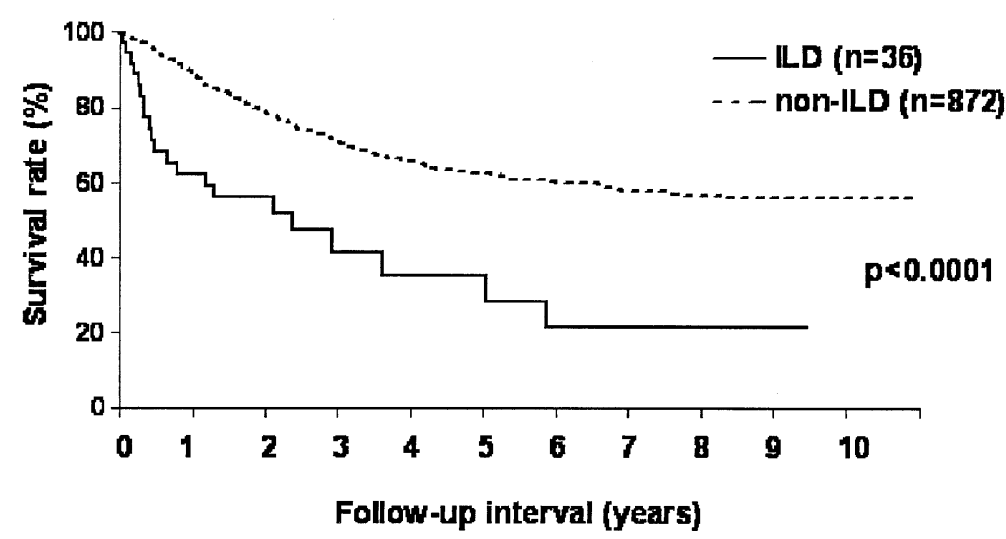

Figure 1. Overall survival after pulmonary resection for lung cancer. The 5-year survivals for the non-ILD group (n $=872$, loss of 23 patient follow-up) and the ILD group $(n=36)$ were $62.5 \%$ and $35.6 \%$, respectively. A statistically significant difference was found between the non-ILD and the ILD $(\log -\operatorname{rank} P<.0001)$.

operative indication for lung cancer, we should take into account the influence of ILD and other risk factors on prognosis.

This retrospective study has certain limitations and biases. First, only selected ILD patients who were thought to tolerate operation were included in this study. Patients who had severe impairment of pulmonary function were excluded from operative indication. Patients who need pneumonectomy or extended resection were also excluded, even though they had permissive predicted postoperative pulmonary function. This selection bias might have a significant impact on survival. Second, there was a possibility that incomplete or limited resection was required in some ILD patients because of impaired pulmonary function. However, a few limited resections were performed in the ILD group, consistent with that in the non-ILD group. This was because pulmonary function was relatively well preserved and limited resection was technically inadequate because of the tumor size. Third, because an inadequate number of ILD patients were compared with a larger number of non-ILD patients, statistical significance may not be obtained in some analyses.

Finally, our results suggest that a strategy for preventing postoperative IP should be established for improving short- and long-term survival. It has been reported that prophylactic chest physiotherapy reduced the incidence of pulmonary complications after major abdominal surgery from $27 \%$ to $6 \% .{ }^{14}$ Some patients with ILD might be responsive to steroid therapy. ${ }^{15}$ Therefore, we have been challenging prophylactic treatment, including immediate preoperative steroid administration, perioperative aggressive pulmonary rehabilitation, and restriction of fluid administration to reduce the risk of pneumonia, lung edema, and interstitial pneumonia.

In conclusion, ILD was a risk factor that increased postoperative morbidity and mortality in patients with lung
TABLE 4. Causes of deaths

\begin{tabular}{|c|c|c|c|}
\hline & $\begin{array}{c}\text { Non-ILD } \\
(n=895)\end{array}$ & $\begin{array}{c}\text { ILD } \\
(n=36)\end{array}$ & $P$ value \\
\hline Deaths & $292(32.6 \%)$ & $21(58.3 \%)$ & 0.0020 \\
\hline Primary cancer & $215(73.6 \%)$ & $11(52.4 \%)$ & \\
\hline Respiratory failure & $27(9.3 \%)$ & $7(33.3 \%)$ & 0.0028 \\
\hline Others & $50(17.1 \%)$ & $3(14.3 \%)$ & \\
\hline
\end{tabular}

TABLE 5. Multivariate analysis of risk factors for overall mortality by proportional hazard model

\begin{tabular}{lcccc}
\hline Variables & \multicolumn{4}{c}{ Hazard } \\
& Chi-square & ratio & 95 \% Cl & P value \\
\hline ILD & 4.683 & 1.903 & $1.012-3.236$ & .031 \\
Gender (male) & 13.41 & 1.766 & $1.304-2.401$ & .0003 \\
Age & 10.57 & 1.015 & $1.008-1.027$ & .001 \\
PSH & 7.140 & 1.511 & $1.137-2.084$ & .008 \\
Operation method & 13.42 & & & .001 \\
$\quad$ Pneumonectomy & & 1 & reference & \\
$\quad$ Lobectomy & 1.367 & 0.784 & $0.521-1.179$ & .242 \\
$\quad$ Segmentectomy/ & 3.052 & 1.649 & $0.941-2.892$ & .081 \\
$\quad$ partial resection & & & & \\
Pathologic stage & 23.08 & & & $<.0001$ \\
$\quad$ I & & 1 & reference & \\
$\quad$ II & 2.854 & 1.244 & $0.966-1.602$ & .091 \\
$\quad$ III & 14.52 & 1.498 & $1.217-1.844$ & .0001 \\
$\quad$ IV & 13.76 & 2.573 & $1.561-4.238$ & .0002 \\
Neoadjuvant/adjuvant & 2.193 & 0.827 & $0.643-1.064$ & .139 \\
$\quad$ therapy & & & & \\
\hline
\end{tabular}

$\mathrm{Cl}$, Confidence interval; ILD, interstitial lung disease; $B M I$, body mass index; $P S H$, positive smoking history.

cancer. Even though patients with ILD tolerated surgical resection, the long-term survival was still lower due to respiratory failure compared with those without ILD. Consideration should be given to develop more aggressive strat- 
egies for prophylactic management in patients with ILD undergoing pulmonary resection.

We thank Ms Eri Sekine, BS, MPH (Data Management \& Biostatistics, Wyeth Lederle Japan, Tokyo, Japan) for statistical assistance.

\section{References}

1. Tockman MS. Other host factors and lung cancer susceptibility. In: Samet JM, editor. Epidemiology of lung cancer. New York: Marcel Dekker; 1994. p. 397-412.

2. Hanley ME, King TE Jr, Schwarz MI, Watters LC, Shen AS, Cherniak RM. The impact of smoking on mechanical properties of the lungs in idiopathic pulmonary fibrosis and sarcoidosis. Am Rev Respir Dis. 1991;144:1102-6.

3. Samet JM, Humble CG, Pathak DR. Personal and family history of respiratory disease and lung cancer risk. Am Rev Respir Dis. 1986; 134:466-70.

4. Flaherty KR, Martinez FJ. The role of pulmonary function testing in pulmonary fibrosis. Curr Opin Pulm Med. 2000;6:404-10.

5. Martinod E, Azorin JF, Sadoun D, et al. Surgical resection of lung cancer in patients with underlying interstitial lung disease. Ann Thorac Surg. 2002;74:1004-7.
6. Doherty MJ, Pearson MG, O'Grady EA, Pellegrini V, Calverley PM. Cryptogenic fibrosing alveolitis with preserved lung volumes. Thorax. 1997;52:998-1002.

7. Matsushima H, Tanaka S, Saiki Y, et al. Lung cancer associated with usual interstitial pneumonia. Pathol Int. 1995;45:925-32.

8. Turner-Warwick M, Courtenay Evans R. Pulmonary manifestations of rheumatoid disease. Clin Rheum Dis. 1977;3:594-604.

9. Patterson CD, Harville WE, Pierce JA. Rheumatoid lung disease. Ann Intern Med. 1965;62:685-90.

10. Roschmann RA. Pulmonary fibrosis in rheumatoid arthritis: a review of clinical features and therapy. Semin Arthritis Rheum. 1987;16:17485 .

11. King TE Jr, Schwarz MI, Brown K, et al. Idiopathic pulmonary fibrosis: relationship between histopathologic features and mortality. Am J Respir Crit Care Med. 2001;164:1025-32.

12. Lamblin C, Bergoin C, Saelens T, Wallaert B. Interstitial lung diseases in collagen vascular diseases. Eur Respir J Suppl. 2001;32:69s-80s.

13. Battafarano RJ, Piccirillo JF, Meyers BF, et al. Impact of comorbidity on survival after surgical resection in patients with stage I non-small cell lung cancer. $J$ Thorac Cardiovasc Surg. 2002;123:280-7.

14. Fagevik Olsen M, Hahn I, Nordgren S, Lonroth H, Lundholm K. Randomized controlled trial of prophylactic chest physiotherapy in major abdominal surgery. Br J Surg. 1997;84:1535-8.

15. Flaherty KR, Toews GB, Lynch JP 3rd, et al. Steroids in idiopathic pulmonary fibrosis: a prospective assessment of adverse reactions, response to therapy, and survival. Am J Med. 2001;110:278-82. 